\title{
Response of Triple Negative Breast Cancer to Neoadjuvant Chemotherapy: Correlation between Ki-67 Expression and Pathological Response
}

\author{
Gamal M Elnemr ${ }^{1,2 *}$, Ahmed H El-Rashidy ${ }^{3,4}$, Ahmed H Osman ${ }^{4,5}$, Lotfi F Issa ${ }^{6,7}$, \\ Osama A Abbas ${ }^{8,9}$, Abdullah S Al-Zahrani ${ }^{10}$, Sheriff M El-Seman ${ }^{10,11}$, Amrallah \\ A Mohammed ${ }^{12}$, Abdelghani A Hassan ${ }^{13,14}$
}

\begin{abstract}
Triple-negative breast cancers constitute about $15 \%$ of all cases, but despite their higher response to neoadjuvant chemotherapy, the tumors are very aggressive and associated with a poor prognosis as well as a higher risk of early recurrence. This study was retrospectively performed on 101 patients with stage II and III invasive breast cancer who received 6-8 cycles of neo-adjuvant chemotherapy. Out of the total, 23 were in the triple negative breast cancer subgroup. Nuclear Ki-67 expression in both the large cohort group $(n=101)$ and triple negative breast cancer subgroup $(\mathrm{n}=23)$ and its relation to the pathological response were evaluated. The purpose of the study was to identify the predictive value of nuclear protein Ki-67 expression among patients with invasive breast cancers, involving the triple negative breast cancer subgroup, treated with neoadjuvant chemotherapy in correlation to the rate of pathological complete response. The proliferation marker Ki-67 expression was highest in the triple negative breast cancer subgroup. No appreciable difference in the rate of Ki-67 expression in triple negative breast cancer subgroup using either a cutoff of $14 \%$ or $35 \%$. Triple negative breast cancer subgroup showed lower rates of pathological complete response. Achievement of pathological complete response was significantly correlated with smaller tumor size and higher Ki-67 expression. The majority of triple negative breast cancer cases achieved pathological partial response. The study concluded that Ki-67 is a useful tool to predict chemosensitivity in the setting of neoadjuvant chemotherapy for invasive breast cancer but not for the triple negative breast cancer subgroup.
\end{abstract}

Keywords: Triple negative breast cancer - neoadjuvant chemotherapy - Ki-67 - pathological response

Asian Pac J Cancer Prev, 17 (2), 807-813

\section{Introduction}

Breast cancer is considered as a heterogeneous disorder whose types are variable biologically and associated with various clinical prognoses and therapeutic responses (Burstein et al., 2008). Intrinsic molecular variants of breast cancer, namely luminal A, luminal B, HER-2 positive and basal-like, were found in a complementary DNA microarray study performed on 65 different breast tumors to analyze about 500 genes (Sorlie et al., 2001).

These breast cancer variants were commonly approximated by using routine markers into the following categories that possessed various prognoses; luminal A: ER+ and/or PR+/HER2-; luminal B: ER+ and/or PR+/ HER2+; HER-2 positive: ER-/PR-/HER2+ and basal-like (basaloid or triple-negative) ER-/PR-/HER2- and/or $\mathrm{CK} 5 / 6+$ and/or EGF+ (epidermal growth factor-positive) (Burstein et al., 2008).

TNBC means invasive breast cancer lacking hormonal (estrogen and progesterone) receptor (ER/PR) as well as HER2 (ERBB2) expression. It represents $15 \%$ of total invasive breast cancers, occurs at a higher rate in young African-American women, and being in general of a higher grade and most of them show a signature of

\footnotetext{
${ }^{1}$ Department of Internal Medicine, ${ }^{4}$ Department of Pathology, ${ }^{7}$ Department of Community Medicine, ${ }^{13}$ Department of Physiology, Faculty of Medicine, Taif University, ${ }^{9}$ Department of Medicine, King Abdul-Aziz Hospital, Taif, ${ }^{10}$ Oncology Center, King Abdullah Medical City, Holly Capital, Saudi Arabia, ${ }^{2}$ Department of Medical and Radiological Research, Nuclear Materials Authority, ${ }^{3}$ Department of Pathology, Faculty of Medicine, Al-Azhar University (Assuit Branch), ${ }^{5}$ Department of Pathology, Faulty of Veterinary Medicine, Cairo University, ${ }^{6}$ Department of Community Medicine, Faculty of Medicine, Al-Azhar University, ${ }^{8}$ Department of Clinical Oncology, Faculty of Medicine, Ain-Shams University, ${ }^{11}$ Department of Medical Oncology, Faculty of Medicine, Mansura University, ${ }^{12}$ Department of Medical Oncology, Faculty of Medicine, Zagagzig University, ${ }^{14}$ Department of Physics, Faculty of Science, Zagagzig University, Egypt*For correspondence:asmrt1711@yahoo.com
} 
basaloid gene expression (Mayer et al., 2014).

Triple negative and basal-like breast cancers are actually different entities with about $30 \%$ discordance, across several studies (Anders and Carey, 2009). However, for ease, basal-like breast cancers have been referred as triple negative breast cancer (Berrada et al., 2010).

Because of the aggressive histopathological findings seen in TNBC than other breast cancer variants, such as higher grades and higher mitotic index, TNBC is accompanied by increased rate of early recurrence as well as a distant blood spread, therefore, it possesses an overall poor prognosis (Peddi et al., 2011).

Neoadjuvant chemotherapy means intervention with chemotherapeutic drugs before starting surgical treatment aiming at decreasing the size of breast cancer mass, allowing the planned surgical procedure (Von Minckwitz and Martin, 2012).

Pathologic complete response (pCR) is a defined as the absence of residual invasive tumor tissue from both breast and axilla after neoadjuvant chemotherapy. Many clinical studies of neoadjuvant chemotherapy have established a decreased cancer recurrence rate and a favorable longterm prognosis in patients achieving $\mathrm{pCR}$ to neoadjuvant treatment rather than those harboring residual tumor tissues after therapy (Burstein et al., 2008).

Although many clinical trials stated that TNBC possessed a better response to neoadjuvant chemotherapy with higher pCR rate than other breast cancer variants, however, more than fifty percent of patients with TNBC tumors didn't attain a pCR and demonstrated worse outcomes (Von Minckwitz and Martin, 2012).

The risk of breast cancer recurrence has been predicted by cell proliferation markers (Milde-Langosch et al., 2013). Among these proliferation markers is the nuclear protein $\mathrm{Ki}-67$ that is detected easily by immunohistochemical techniques (Inwald et al., 2013). All phases of the cell proliferation cycle, apart from G0 (quiescent) phase, express Ki-67 (Gerdes et al., 1991). Several neoadjuvant series have investigated the predictive and prognostic values of Ki-67 in breast cancer patients. Breast cancer with high Ki-67 expression, responds better to chemotherapy, but is associated with poor prognosis. This phenomenon is similar to the triple negative paradox. In addition, TNBC is associated with a higher expression of Ki-67 than non-TNBC (Keam et al., 2011). However, there was neither standard procedure nor generally accepted Ki-67 definition (Yerushalmi et al., 2010; Luporsi et al., 2012).

The aims of this study were to assess the predictive value of the nuclear protein $\mathrm{Ki}-67$ expression among patients with invasive breast cancers, including a TNBC subgroup, treated with neoadjuvant chemotherapy in correlation to the rate of $\mathrm{pCR}$.

\section{Materials and Methods}

\section{Eligibility}

This is a retrospective study. Between February 2012 and February 2014, at the Oncology Center of King Abdullah Medical City (KAMC), Makkah, KSA, patients aged $>25$ years old, with stage II and III invasive breast cancers, according to the Joint Committee on Cancer (AJCC), 7th edition (2010) staging system, $(n=101)$ received neoadjuvant chemotherapy. Patients with objectively measured mass and had adequate performance status (ECOG $=0-2$ ) were included while those undergoing a prior chemotherapy or having an inadequate bone marrow, hepatic, cardiac and/or renal functions were excluded.

\section{Data collection, ethical aspect}

This study was approved by the Ethics Committee of KAMC, Makkah. The intervention applied did not represent any more than minimal harm to the recruited patients; consequently, no informed consent had been obtained. Human subject's names had kept in a password protected database and only had been linked with the identification of this research. There were no patient identifiers.

Histodiagnosis, clinical staging and evaluation of clinical response

All invasive breast cancer patients were diagnosed pathologically through core needle biopsy. Clinical staging work-up included CT chest, abdomen \& pelvis, together with bone scintigraphy that was done to rule out stage IV breast cancer. Baseline imaging (enhanced both MRI breast and CT chest/axilla) was performed 1-2 weeks before starting neoadjuvant chemotherapy. Moreover, breast cancer was staged according to the American Joint Committee on Cancer (AJCC), 7th edition (Edge et al., 2010).

Radiological tumor response was also evaluated using enhanced both MRI breast and CT chest/axilla according to RECIST criteria as follows; complete response (CR) was termed as complete disappearance of all assessable lesions; partial response (PR) that defined as a $>30 \%$ reduction in the sum of the longest diameters of all measurable masses; stable disease (SD) that meant a $<30 \%$ reduction or a $<20 \%$ increase in the sum of the longest diameters of all measurable lesions; and progressive disease (PD) which detected $>20 \%$ increase in the area (s) of original measurable lesion or appearance of a new lesion.

\section{Immunohistochemistry technique}

Immunohistochemistry (IHC) was performed on the specimens that were histopathologically confirmed as invasive breast cancer and obtained before starting treatment by core needle biopsy. Estrogen receptor (ER), progesterone receptor (PR), human epidermal growth factor receptor-2 (HER-2) and Ki-67 expressions were evaluated. ER and PR positivity was defined as $\geq 10 \%$ positive tumor cells with nuclear staining. HER-2 positivity was scored as $3+$ by IHC, or positive FISH (fluorescent in situ hybridization) for those with $2+$ by IHC. Cells stained for Ki-67 were counted and expressed as a percentage that was determined by the number of Ki-67 positive cells among the total number of counting tumor cells. High expression of Ki-67 was defined as $\geq 14 \%$ stained cells counted in 3 high power fields. A cutoff of $\geq 14 \%$ provided the best prognosis-prediction results in 
our institute. The intensity of staining was graded as very weak, mild, moderate and marked nuclear immunopositive reaction. Another cutoff of $35 \%$ was tested to compare the expected results.

TNBC was defined as the tumor that was ER (-), PR (-) and HER-2 (-) regardless the expression of epidermal growth factor receptor (EGFR) and basal cytokeratins.

\section{Evaluation of pathologic response}

Specimens without a residual invasive tumor in both the breast and the regional lymph nodes were classified as a pathological complete response (pCR). Residual ductal carcinoma in situ was also included in the pCR category. Otherwise the specimens that did not achieve pCR category were classified as residual disease.

\section{Treatment protocol}

The chemotherapy regimen was anthracycline/ taxane-based for six to eight cycles. Granulocyte colony stimulating factor (G-CSF) was offered as primary prophylaxis if needed. Adequate bone marrow, liver and kidney functions were assessed 1 day prior to each cycle of the neoadjuvant chemotherapy. After completion of the treatment, the patients were subjected to primary surgery either breast conservation surgery (BCS) or modified radical mastectomy (MRM).

\section{Statistical analysis}

Patient characteristics prior to neoadjuvant chemotherapy were presented with descriptive statistics. Data will be analyzed in KAMC Oncology Center, Research Unit. The Chi-square test (Fisher's exact test) was carried out for comparison between groups regarding their quantitative outcome. Routine clinicopathological predictive and prognostic parameters in breast cancer as well as their association and correlation with the expressed Ki-67 and also with the response to neoadjuvant chemotherapy were concentrated on.

\section{Results}

Between February 2012 and February 2014, one hundred and one $(n=101)$ patients with invasive breast cancer, stage II-III received neoadjuvant chemotherapy, and were assessed for the study protocol (Figure 1). Of the 101 patients listed in this trial, 23 were of TNBC subtype. Patient clinicopathological characteristics of the whole cohort (mother group) $(n=101)$ and TNBC subgroup $(n=23)$ prior to the neoadjuvant chemotherapy were shown in Table 1.

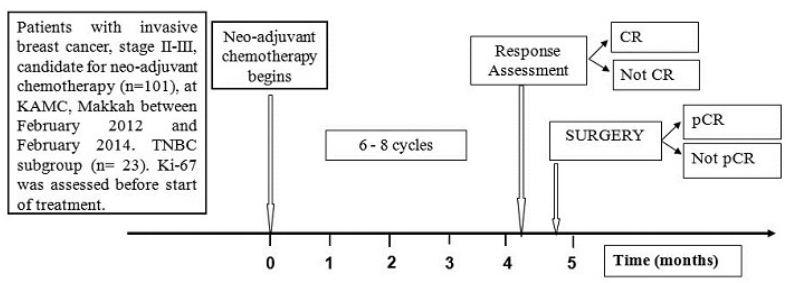

Figure 1. Treatment Design. KAMC: King Abdullah Medical City, TNBC: triple negative breast cancer, CR: complete response (clinical), pCR: pathological complete response
Table 1. Patient Clinicopathological Characteristics of the Whole Cohort (Mother Group) and TNBC Subgroup

\begin{tabular}{|c|c|c|c|c|}
\hline \multirow[t]{2}{*}{ Parameters } & \multicolumn{2}{|c|}{ Whole cohort } & \multicolumn{2}{|c|}{ TNBC subgroup } \\
\hline & N (101) & $\%$ & $\mathrm{~N}(23)$ & $\%$ \\
\hline \multicolumn{5}{|l|}{ Age (years) } \\
\hline$<40$ & 20 & 19.8 & 4 & 17.4 \\
\hline$>40$ & 81 & 80.2 & 19 & 82.6 \\
\hline \multicolumn{5}{|l|}{ Menopause } \\
\hline Premenopause & 60 & 59.4 & 13 & 56.5 \\
\hline Postmenopause & e 41 & 40.6 & 10 & 43.5 \\
\hline \multicolumn{5}{|l|}{ Grade } \\
\hline 1 & 4 & 4 & 0 & 0 \\
\hline 2 & 56 & 55.4 & 6 & 26.1 \\
\hline 3 & 41 & 40.6 & 13 & 73.9 \\
\hline \multicolumn{5}{|c|}{ Multicentric tumors } \\
\hline Yes & 23 & 22.8 & 4 & 17.4 \\
\hline No & 78 & 77.2 & 19 & 82.6 \\
\hline \multicolumn{5}{|l|}{ LVI } \\
\hline Yes & 46 & 45.5 & 12 & 52.2 \\
\hline No & 55 & 54.5 & 11 & 47.8 \\
\hline \multicolumn{5}{|l|}{ Tumor stage } \\
\hline $\mathrm{T} 1$ & 6 & 5.9 & 1 & 4.3 \\
\hline $\mathrm{T} 2$ & 31 & 30.7 & 6 & 26.1 \\
\hline $\mathrm{T} 3$ & 40 & 39.6 & 11 & 47.8 \\
\hline $\mathrm{T} 4$ & 24 & 23.8 & 5 & 21.7 \\
\hline \multicolumn{5}{|l|}{ *Ki-67 } \\
\hline Low & 25 & 24.8 & 2 & 8.7 \\
\hline High & 76 & 75.2 & 21 & 91.3 \\
\hline \multicolumn{5}{|l|}{ ER/PR status } \\
\hline Negative & 33 & 32.7 & 23 & 100 \\
\hline Positive & 68 & 67.3 & 0 & 0 \\
\hline \multicolumn{5}{|l|}{ HER-2 } \\
\hline Negative & 77 & 76.2 & 23 & 100 \\
\hline Positive & 24 & 23.8 & 0 & 0 \\
\hline
\end{tabular}

LVI, lymphovascular invasion; ER, estrogen receptor; PR, progesterone receptor; HER2, human epidermal growth factor receptors type 2. *High Ki-67 expression was defined as $\geq 14 \%$

Table 2. Other TNBC Subgroup Clinic-pathologic Characteristics

\begin{tabular}{lcr}
\hline Parameters & \multicolumn{2}{c}{ TNBC subgroup } \\
\cline { 2 - 3 } & $\mathrm{N}(23)$ & $\%$ \\
\hline Pathological subtype & 22 & 95.7 \\
$\quad$ IDC & 1 & 4.3 \\
$\quad$ ILC & 17 & 73.9 \\
Presence of DCIS (ductal carcinoma in-situ) & \\
$\quad$ No & 6 & 26.1 \\
$\quad$ Yes & & \\
Body Mass Index (BMI) & 2 & 8.7 \\
18 - <25 & 8 & 34.8 \\
$25-30$ & 12 & 52.2 \\
$>30-40$ & 1 & 4.3 \\
$>40$ & & \\
*Ki-67(35\%) & 3 & 13 \\
Low & 20 & 87 \\
High & & \\
\hline
\end{tabular}

TNBC: triple negative breast cancer; IDC: invasive duct carcinoma; ILC: invasive lobular carcinoma; *High Ki-67 expression was defined using a higher cutoff $\geq 35 \%$

Most patients $80.2 \%$ were at the age of 40 years or older, either in the whole cohort or TNBC subgroup. However, in both mother group and TNBC subgroup, 

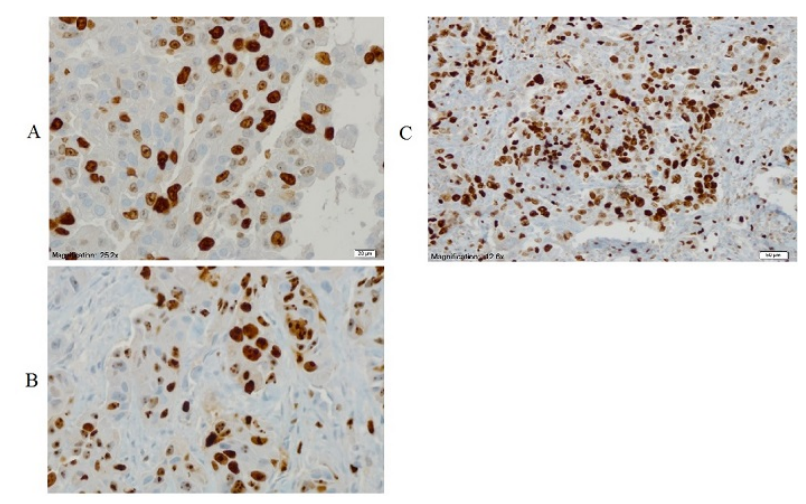

Figure 2. Photomicrographs of Triple Negative Breast Cancers (TNBC) showing Moderate (A), Moderate to Marked (B) and Marked (C) Ki-67 Expression of Tumor Cells [Brownish Nuclear Immune-staining]. Immunohistochemistry X 200 (for $A$ and B) and X 100 (for C)

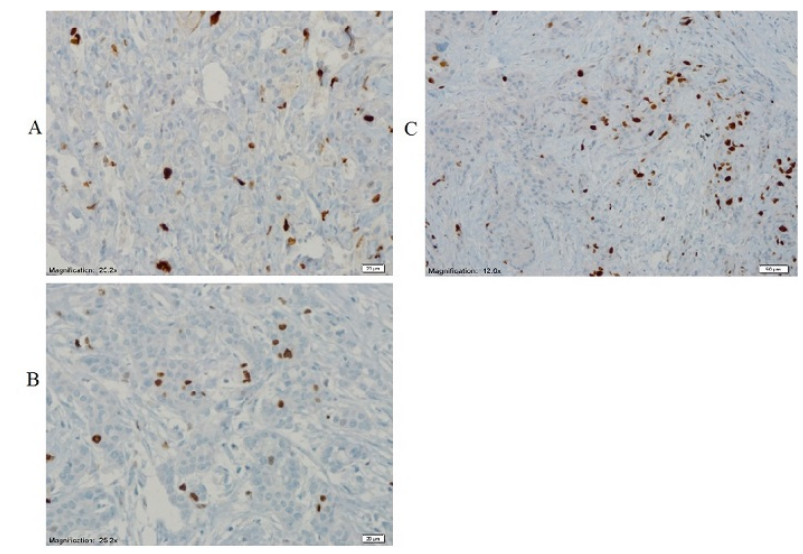

Figure 3. Photomicrographs of Invasive Duct Carcinoma showing Very Weak (A and B) and Mild (C) Ki-67 Expression of Tumor Cells (Brownish Nuclear Immune-staining). Immunohistochemistry X 100

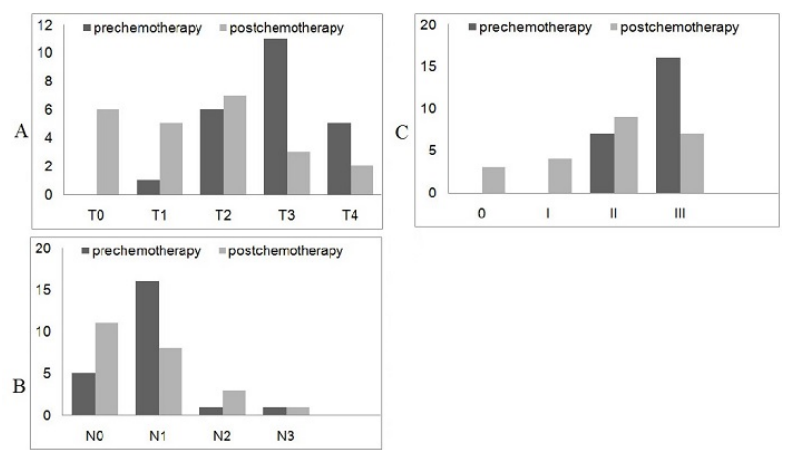

Figure 4. Clinical (Radiological) Response of Tumor size (T) (A), Nodal Stage (N) (B) andTumor TNM Stage (C) of TNBC Patients $(n=23)$ Received Neoadjuvant Chemotherapy in KAMC, Makkah, KSA between February 2012 and February 2014

premenopausal women represented the largest component being $59.4 \%$ and $56.5 \%$ respectively.

Considering tumor characteristics shown in
Table 4. Comparison of Pathological Complete Response (pCR) Versus non-pCR in the whole Cohort and TNBC Subgroup

\begin{tabular}{lcccc}
\hline Parameters & \multicolumn{2}{c}{ Whole cohort } & \multicolumn{2}{c}{ TNBC subgroup } \\
\cline { 2 - 5 } & $\mathrm{n}(101)$ & $\%$ & $\mathrm{n}(23)$ & $\%$ \\
\hline pCR & 25 & 24.7 & 3 & 13 \\
non- pCR & 76 & 75.3 & 20 & 87 \\
\hline
\end{tabular}

Table 5. Detailed Pathologic Response in TNBC Subgroup

\begin{tabular}{lcc}
\hline Type of pathological response & \multicolumn{2}{c}{ TNBC subgroup } \\
\cline { 2 - 3 } & $\mathrm{n}(23)$ & $\%$ \\
\hline Pathological complete response (pCR) & 3 & 13 \\
Pathological partial response (pPR) & 15 & 65.2 \\
Pathological stable response (pSD) & 5 & 21.7 \\
\hline
\end{tabular}

Table 6. TNM Clinical Down Staging in TNBC Subgroup after Neoadjuvant Chemotherapy

\begin{tabular}{lcccc}
\hline TNM stage & \multicolumn{2}{c}{ Prechemotherapy } & \multicolumn{2}{c}{ Postchemtherapy } \\
\cline { 2 - 5 } & $\mathrm{n}$ & $\%$ & $\mathrm{n}$ & $\%$ \\
\hline Tumor size (clinical) & & & & \\
T0 & 0 & 0 & 6 & 26.1 \\
T1 & 1 & 4.3 & 5 & 21.7 \\
T2 & 6 & 26.1 & 7 & 30.4 \\
T3 & 11 & 47.8 & 3 & 13 \\
T4 & 5 & 21.7 & 2 & 8.7 \\
Nodal stage (clinical) & & & & \\
N0 & 5 & 21.7 & 11 & 47.8 \\
N1 & 16 & 69.6 & 8 & 34.8 \\
N2 & 1 & 4.3 & 3 & 13 \\
N3 & 1 & 4.3 & 1 & 4.3 \\
Tumor stage (clinical) & & & \\
0 & 0 & 0 & 3 & 13 \\
I & 0 & 0 & 4 & 17.4 \\
II & 7 & 30.4 & 9 & 39.1 \\
III & 16 & 69.6 & 7 & 30.4 \\
\hline
\end{tabular}

prechemotherapy diagnostic core biopsies, the majority of patients harbored grades 2 and 3 tumors as $96 \%$ and $100 \%$ in the mother group and TNBC subgroup respectively. However, grade 3 histology was more common in the TNBC subgroup, $73.9 \%$ versus $40.6 \%$ in the large cohort group.

Multicentric tumors represented a minority in both groups being $22.8 \%$ and $17.4 \%$ respectively. Lymphovascular invasion (LVI) represented a consistent finding in both groups to be $45.5 \%$ and $52.2 \%$ respectively. Tumor size (T) was also comparable between the two groups that revealed; in both, that the majority of cases were large (T3 and T4). The proliferation marker Ki-67 expression was relatively higher in the TNBC subgroup (91.3\%; Fig (2) than the mother group (75.2\%; Figs.3) using a cutoff of $14 \%$ in its immunostaining. Hormonal receptors were positive in $67.3 \%$ of the mother group, while HER $2 /$ neu was overexpressed in only $23.8 \%$ of the mother group

The majority of TNBC had a high body mass index 
Table 3. Correlation Between The Rate Of Pathological Complete Response (pCR) vs Non-pCR in the Large Cohort $(n=101)$ and different clinicopathological parameters

\begin{tabular}{|c|c|c|c|c|}
\hline Parameters & $\mathrm{pCR}(\%)$ & No pCR (\%) & Total & $\mathrm{P}$ \\
\hline \multicolumn{5}{|l|}{ Age } \\
\hline$<40$ & $4(20.0)$ & $16(80.0)$ & 20 & \multirow[t]{4}{*}{0.83} \\
\hline $40-55$ & $14(21.5)$ & 42 (78.5) & 56 & \\
\hline$>55$ & $7(28.0)$ & $18(72.0)$ & 25 & \\
\hline Total & 25 & 76 & 101 & \\
\hline \multicolumn{5}{|l|}{ Menopause } \\
\hline Premenopause & $15(25.0)$ & $45(75.0)$ & 60 & \multirow[t]{3}{*}{0.94} \\
\hline Postmenopause & $10(24.4)$ & $31(75.6)$ & 41 & \\
\hline Total & 25 & 76 & 101 & \\
\hline \multicolumn{5}{|l|}{ Grade } \\
\hline 1 & $1(25.0)$ & $3(75.0)$ & 4 & \multirow[t]{4}{*}{0.92} \\
\hline 2 & $13(23.2)$ & $43(76.8)$ & 56 & \\
\hline 3 & $11(26.8)$ & $30(73.2)$ & 41 & \\
\hline Total & 25 & 76 & 101 & \\
\hline \multicolumn{5}{|l|}{ Multicentric tumors } \\
\hline Yes & $5(21.7)$ & $18(78.3)$ & 23 & \multirow[t]{3}{*}{0.7} \\
\hline No & 20 (25.6) & $58(74.4)$ & 78 & \\
\hline Total & 25 & 76 & 101 & \\
\hline \multicolumn{5}{|l|}{ LVI } \\
\hline Yes & $8(17.4)$ & $38(82.6)$ & 46 & \multirow[t]{3}{*}{0.12} \\
\hline No & $17(30.9)$ & $38(69.1)$ & 55 & \\
\hline Total & 25 & 76 & 101 & \\
\hline \multicolumn{5}{|l|}{$\mathrm{Ki}-67(14 \%)$} \\
\hline $\mathrm{T} 1$ & $5(83.3)$ & $1(16.7)$ & 6 & \multirow{6}{*}{0.013} \\
\hline $\mathrm{T} 2$ & $5(16.1)$ & $26(83.9)$ & 31 & \\
\hline $\mathrm{T} 3$ & $10(25.0)$ & $30(75.0)$ & 40 & \\
\hline $\mathrm{T} 4$ & $5(20.8)$ & $19(79.2)$ & 24 & \\
\hline Total & 25 & 76 & 101 & \\
\hline Low & $4(16.0)$ & $21(84.0)$ & 25 & \\
\hline High & $21(27.6)$ & $55(72.4)$ & 76 & \multirow[t]{2}{*}{0.016} \\
\hline Total & 25 & 76 & 101 & \\
\hline \multicolumn{5}{|l|}{ ER/PR status } \\
\hline Negative & $5(15.2)$ & $28(84.8)$ & 33 & \multirow[t]{3}{*}{0.12} \\
\hline Positive & $20(29.4)$ & $48(70.6)$ & 68 & \\
\hline Total & 25 & 76 & 101 & \\
\hline \multicolumn{5}{|l|}{ HER-2 } \\
\hline Negative & $16(20.8)$ & $61(79.2)$ & 77 & \multirow[t]{3}{*}{0.097} \\
\hline Positive & $9(37.5)$ & $15(62.5)$ & 24 & \\
\hline Total & 25 & 76 & 101 & \\
\hline
\end{tabular}

pCR: Pathological complete Response, LVI, lymphovascular space invasion; ER, estrogen receptor; PR, progesterone receptor; HER-2, human epidermal growth factor receptor type- 2

(BMI > 25) (Table 2). The predominant histology in TNBC was invasive ductal carcinoma $(n=22,95.7 \%)$ rather than lobular carcinoma $(n=1,4.3 \%)$ while duct carcinoma is situ (DCIS) was found in $26.1 \%$ of TNBC patients. No appreciable differences in low or high Ki-67 expression in TNBC subgroup using either the cutoff $14 \%$ (mentioned in Table 1 that were $8.7 \%$ and $91.3 \%$ respectively) or $35 \%$ (found in Table 2 that were 13\% and $87 \%$ respectively).

There was a significant correlation of the rate of pCR in the large cohort $(n=101)$, to both smaller tumor size $(\mathrm{p}=0.013)$ and the higher Ki-67 expression $(\mathrm{p}=$ 0.016) (Table 3). Patient's age, menopausal status, tumor multicentricity, lymphovascular invasion and hormonal as well as HER-2 receptor expression status had no significant impact on achieving pCR.

The higher $\mathrm{pCR}$ rate was achieved in the whole cohort
(25 cases, $24.7 \%$ ) than in the TNBC subgroup ( 3 cases, $13 \%$ ) (Table 4). However, $65.2 \%$ of patients in the TNBC subgroup achieved a pathological partial response (pPR) (Table 5). Detailed clinical response and tumor down staging of TNBC subgroup were shown in Table 6 and Figs. (4).

\section{Discussion}

In the present study, we found that rate of $\mathrm{pCR}$ to neoadjuvant chemotherapy was $24.7 \%(n=25)$ in the whole cohort $(n=101)$. This finding was consistent with the range of $\mathrm{pCR}$ rates to prospective randomized trials of preoperative chemotherapy of 15\%-40\% mentioned in the literature (Burstein et al., 2008).

Moreover, high Ki-67 expression (cutoff $>14 \%$ ) was significantly correlated with achieving a pCR in the large cohort ( $\mathrm{p}=0.016$ ). This result matched with the finding of several studies that reported that high Ki-67 expression was associated with higher response rates to neoadjuvant chemotherapy in breast cancer (Keam et al., 2011).

Also, our study revealed that TNBC patients accounted for about $23 \%$ of the whole cohort of invasive duct carcinomas and this approximated to the percentage mentioned by Aysola et al., who stated that TNBC represented up to $20 \%$ of invasive breast cancers (Aysola et al., 2013).

More than half of our studied TNBC patients were premenopause and more than $90 \%$ had a high body mass index (BMI). These findings were matched with the epidemiologic description of TNBC in the literature (Aysola et al., 2013).

Furthermore, the majority of our TNBC patients had an invasive ductal carcinoma (95.7\%), high Ki-67 expression ( $91.3 \%$ or $87 \%$ when at cutoff of $14 \%$ or $35 \%$ was used, respectively), and had a high histological grade. These findings were consistent with the histological TNBC criteria in the literature (Gluz et al., 2009). Surprisingly, our work revealed that TNBC subgroup achieved a lower pCR rate (13\%) compared to the large cohort (24.7\%) in contrast to the historical reports of higher $\mathrm{pCR}$ rates in TNBC than for other breast tumor types in the neoadjuvant setting. However, $65.2 \%$ of our TNBC cases achieved pathologic partial response.

Historically, TNBC had responded well in the neoadjuvant setting, with high rates of $\mathrm{pCR}$ as reported by von Minckwitz and Martin (2012). Also, Liedtke et al. (2008) detected a pCR rate of $22 \%$ in TNBC patients versus $11 \%$ in $\mathrm{ER}+$ invasive breast cancers in 1118 patients, of which 225 patients had TNBC (23\%) who received neoadjuvant anthracycline and taxane based chemotherapy regimens. Similarly, Liu et al. (2013) gave 7 cycles of platinum-based neo-adjuvant chemotherapy in 717 patients, of whom $31 \%$ were TNBC, and found that the clinical complete response (cCR) rate and the pathological complete response (pCR) rates were significantly higher in TNBC group than in non-TNBC one.

However, the low pCR rate achieved by our TNBC cases was close to that reported in a small study at Dana Farber Cancer Institute, where 26 TNBC patients received six cycles of neoadjuvant single-agent cisplatin, and only 
$4(15 \%)$ achieved complete response (CR), after exclusion of two cases with BRCA mutation (Silver et al., 2010). Similarly, Bidard et al., reported a pCR rate of $17 \%$ in a relatively large cohort of TNBC group $(n=120)$ receiving a combined neoadjuvant chemotherapy regimen composed of cyclophosphamide, epirubicin and 5-fluorouracil (CEF) for 4 to 6 cycles (Bidard et al., 2008).

Our low pCR rate in TNBC subgroup might be also explained by the type of chemotherapeutic agents that had been used. Rocca and colleagues conducted a retrospective analysis of core biopsies of patients with breast cancer treated with neoadjuvant cisplatin-based chemotherapy in breast cancer and showed that administration of cisplatin without anthracyclines yielded a higher rate of pCR in patients with p63-positive tumors (Rocca et al., 2008).

Again, our low pCR rate in TNBC might be also explained by the heterogeneous genetic nature of TNBC. Gene expression analyses identified six distinct TNBC subtypes, each displaying a unique biology with different clinical outcomes (Mayer et al., 2014). Also, Masuda et al., found that despite the overall pCR rate was $28 \%$ in a retrospective analysis of 130 TNBC cases treated with neoadjuvant combination of doxorubicin, cyclophosphamide and paclitaxel, the genetic subtypespecific responses have differed substantially, being the greatest in the basal like1 (BL1) subtype (52\%) compared to lower rates of the basal like 2 (BL2), with luminal androgen receptor (LAR) and mesenchymal stem like (MSL) subtypes being 0\%, 10\%, and 23\%, respectively (Masuda et al., 2013).

In our study, $87 \%$ of TNBC cases had residual disease. Andre and Zielinski (2012) stated in their review article that the majority of TNBC patients has a residual disease following treatment of early breast cancer, with an expected high risk of relapse and a sharp decrease in their survival within the first 3-5 years in such fraction of TNBC patients with chemoresistant disease unfortunately representing $>50 \%$ of TNBC (Gluz et al., 2009) highlighting a need for more effective neoadjuvant therapies or defined adjuvant, residual disease protocols (Anders and Carey, 2009).

In our TNBC patients, although Ki67 was highly expressed, although this was reflected in the rate of pCR. Our results didn't cope with the results of Keam et al., who studied the predictive and prognostic value of Ki-67 among 105 patients with TNBC treated with neoadjuvant chemotherapy. They found that TNBC with high Ki-67 expression ( $\geq 10 \%$ ) showed a higher $\mathrm{pCR}$ rate to neoadjuvant chemotherapy than TNBC with low Ki67 expression $(\mathrm{P}=0.019)$. None of the low Ki-67 group achieved pCR. They concluded that high proliferation index $\mathrm{Ki}-67$ can be used for further classification of TNBC into two subtypes with different responses and prognosis (Keam et al., 2011).

In conclusion, Ki-67 proliferation marker was detected as a useful and an easy tool to predict chemosensitivity in the neoadjuvant or similar setting of invasive breast cancer. Although Ki-67 was highly expressed in TNBC, this proliferation index could not predict chemosensitivity in such subgroup of invasive breast cancer. Chemotherapy- resistant TNBC remains a medical challenge calling for more efforts to decipher it.

\section{References}

Anders CK, Carey LA (2009). Biology, metastatic patterns, and treatment of patients with triple-negative breast cancer. Clinical Breast Cancer, 9, 73-81.

Andre F, Zielinski C (2012). Optimal strategies for the treatment of metastatic triple-negative breast cancer with currently approved agents. Ann Oncology, 23, 46-51.

Aysola K, Desai A, Welch C, et al (2013). Triple negative breast cancer-an overview. Hereditary Genetics: Current Research, 2013.

Berrada N, Delaloge S, Andre F (2010). Treatment of triplenegative metastatic breast cancer: toward individualized targeted treatments or chemosensitization? Ann Oncol, 21, $30-5$.

Bidard F-C, Matthieu M-C, Chollet P, et al (2008). p53 status and efficacy of primary anthracyclines/alkylating agent-based regimen according to breast cancer molecular classes. Ann Oncol, 19, 1261-5.

Burstein HJ, Harris JR, Morrow M (2008). Malignant tumors of the breast. Cancer: principles and practice of oncology. 8th ed. Philadelphia: Lippincott, Williams \& Wilkins, 1606-54.

Edge SB, Byrd DR, Compton CC, et al (2010). AJCC cancer staging manual, Springer New York.

Gerdes J, Li L, Schlueter C, et al (1991). Immunobiochemical and molecular biologic characterization of the cell proliferation-associated nuclear antigen that is defined by monoclonal antibody Ki-67. Am J Pathol, 138, 867.

Gluz O, Liedtke C, Gottschalk N, et al (2009). Triple-negative breast cancer-current status and future directions. Ann Oncol, 492.

Inwald E, Klinkhammer-Schalke M, Hofstädter F, et al (2013). $\mathrm{Ki}-67$ is a prognostic parameter in breast cancer patients: results of a large population-based cohort of a cancer registry. Breast Cancer Res Treatment, 139, 539-52.

Keam B, Im S-A, Lee K-H, et al (2011). Ki-67 can be used for further classification of triple negative breast cancer into two subtypes with different response and prognosis. Breast Cancer Res, 13, 22.

Liedtke C, Mazouni C, Hess KR, et al (2008). Response to neoadjuvant therapy and long-term survival in patients with triple-negative breast cancer. J Clin Oncol, 26, 1275-81.

Liu M, Mo QG, Wei CY, et al (2013). Platinum-based chemotherapy in triple-negative breast cancer: A meta-analysis. Oncology letters, 5, 983-91.

Luporsi E, Andre F, Spyratos F, et al (2012). Ki-67: level of evidence and methodological considerations for its role in the clinical management of breast cancer: analytical and critical review. Breast Cancer Res Treatment, 132, 895-915.

Masuda H, Baggerly KA, Wang Y, et al (2013). Differential pathologic complete response rates after neoadjuvant chemotherapy among molecular subtypes of triple-negative breast cancer. ASCO Annual Meeting Proc, 1005.

Mayer IA, Abramson VG, Lehmann BD, et al (2014). New strategies for triple-negative breast cancer-deciphering the heterogeneity. Clinical Cancer Res, 20, 782-90.

Milde-Langosch K, Karn T, Müller V, et al (2013). Validity of the proliferation markers Ki67, TOP2A, and RacGAP1 in molecular subgroups of breast cancer. Breast Cancer Res Treatment, 137, 57-67.

Peddi PF, Ellis MJ, Ma C (2011). Molecular basis of triple negative breast cancer and implications for therapy. Int $J$ Breast Cancer, 2012. 
Rocca A, Viale G, Gelber RD, et al (2008). Pathologic complete remission rate after cisplatin-based primary chemotherapy in breast cancer: correlation with p63 expression. Cancer Chemotherapy Pharmacol, 61, 965-71.

Silver DP, Richardson AL, Eklund AC, et al (2010). Efficacy of neoadjuvant Cisplatin in triple-negative breast cancer. $J$ Clin Oncol, 28, 1145-53.

Sorlie T, Perou CM, Tibshirani R, et al (2001). Gene expression patterns of breast carcinomas distinguish tumor subclasses with clinical implications. Proc Natl Acad Sci USA, 98, 10869-74.

Von Minckwitz G, Martin M (2012). Neoadjuvant treatments for triple-negative breast cancer (TNBC). Ann Oncol, 23, 35-9.

Yerushalmi R, Woods R, Ravdin PM, et al (2010). Ki67 in breast cancer: prognostic and predictive potential. Lancet Oncol, 11, 174-83. 